\title{
3D reconstruction of tilted cracks using infrared thermography and the virtual wave concept
}

\author{
by S. Waters*, P. Burgholzer*, A. Mendioroz**, I. Sáez de Ocáriz*** \\ * Research Center for Non Destructive Testing (RECENDT), Altenberger Straße 69, 4040 Linz, Austria, E-mail: \\ peter.burholzer@recendt.at \\ ** Departamento de Física Aplicada I, Escuela Técnica Superior de Ingeniería, Universidad del País Vasco \\ UPV/EHU, Pza. Ingeniero Torres Quevedo 1, 48013 Bilbao, Spain, \\ *** Centro de Tecnologías Aeronáuticas (CTA) Parque Tecnológico de Alava, 01510 Miñano, Spain
}

\begin{abstract}
Within the scope of this work, active thermography is used to assess the ability to detect and image sub-surface planar defects at a range of inclinations. A novel two stage 3D thermographic reconstruction method will be used. This method is able to take advantage of ultrasound reconstruction methods (such as F-SAFT) through the use of virtual waves and the results will be used in combination with experimental data in order to confirm its validity. For each of the experiments, copper slabs of various shapes (triangles, rectangles) are embedded at various angles of inclination in plastic samples.
\end{abstract}

\section{Introduction}

Within the diverse range of non-destructive testing (NDT) methods, infrared (IR) thermography as a wide ranging method has been growing in both popularity and applications. The comprehensive idea of IR thermography is to detect subsurface abnormalities based on a measured surface temperature evolution.

In this research, a method called pulsed phase thermography will be employed due to its ability to merge the advantages of both pulsed and lock-in thermography methods [1]. First used in 1996, through the use of this method, spatial resolution is improved at larger depths in addition to being a fast and reliable technique [2].

For any IR thermography experiment, a reconstruction method is required to enhance the output images to accurately interpret the tested materials properties. There are numerous methods to do this such as TSR (thermographic signal reconstruction), DAC (differential absolute contrast) and the classic approach requiring a sound area [3] [4] [5]. For this research a state-of-the-art method is used which allows advanced ultrasound reconstruction methods to be applied. This is achieved by transforming the data so it behaves like an acoustic signal through the use of virtual waves.

Being able to model the data as an acoustic wave has significant advantage over traditional thermographic reconstruction methods, because unlike irreversible thermal waves, acoustic waves allow time-reversal for reconstruction. Further details of this process are in section 3. This method has been validated by inverting experimental data, where plastic samples containing buried copper slabs of different shapes and inclinations are heated inductively. This method provides accurate characterization of the heat sources generated in the experiments.

\section{Experimental Setup}

During the experiments, defects were created by embedding copper slabs of different shapes and inclinations within 3D printed photopolymer resin (PR48) samples, whose thermal diffusivity is $1.3 \times 10^{-7} \mathrm{~m}^{2} / \mathrm{s}$. The lateral dimensions of the copper slabs are in the $1.7-2.9 \mathrm{~mm}$ range, and the upper side of the slab was buried at various depths $d$ ranging between 300 and $850 \mu \mathrm{m}$ with angles of $0,30,45,60$ and 90 degrees. To improve the emissivity of the plastic samples, the surface is covered with a thin layer of graphite paint. The experimental setup can be seen in Fig. 1, together with a diagram of the samples. During testing, the samples are located in the middle of an $8 \mathrm{~cm}$ diameter induction coil, excited at $120 \mathrm{kHz}$ with a nominal power of $11.2 \mathrm{~kW}$. 


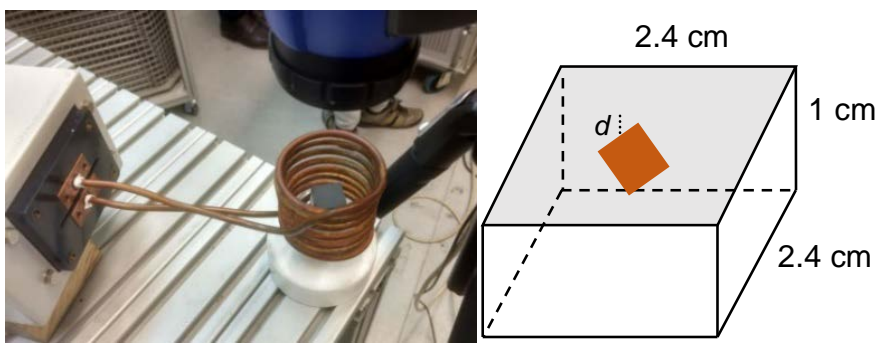

Fig. 1: Experimental eddy current set-up used during testing and sketch of a sample (resin with tilted copper slabs, see text)

\section{3D reconstruction using the virtual wave concept}

One of the most promising thermal reconstruction methods has been set out in [1]. This method involves the use of virtual waves within the reconstruction process and allows all well-known ultrasound methods to be used

To begin with, a 1D reconstruction is performed for every surface pixel. Due to the ill posed nature of thermal diffusion, the regularization method known as the singular value decomposition (SVD) method (for more information please refer to [5]) is used to convert the data for each surface pixel into a virtual wave. The main advantage of using virtual waves is that due to their ability to be described by the wave equation, ultrasound reconstruction methods can be used in this final stage of data processing. These reconstruction methods are highly advanced, mainly because of its longstanding use in medical imaging.

To create the 3D images the frequency domain synthetic aperture focusing technique (F-SAFT) ultrasonic reconstruction method was chosen, but all other ultrasound reconstruction methods are possible. During this process, the F-SAFT algorithm gives an accurate estimation of the initial temperature evolution $\left(T_{0, r e c}(x, y, z)\right)$.

\section{Results}

Based on the simulated data Fig 2 shows the results for a single $2 \mathrm{~mm}$ deep rectangular crack for various angles. For each of the simulations below the shallow end of the crack begins at 300 microns below the surface. The image shows a slice of the 3D reconstructions for 30, 45 and 60 degrees perpendicular to the crack and the sample surface. The dotted line represents the exact shape of the crack $\left(T_{0}\right)$. It can be seen that there is a good correlation between the reconstruction and the exact shape of the crack, although as the crack deepens the reconstruction intensity reduces.

(a)

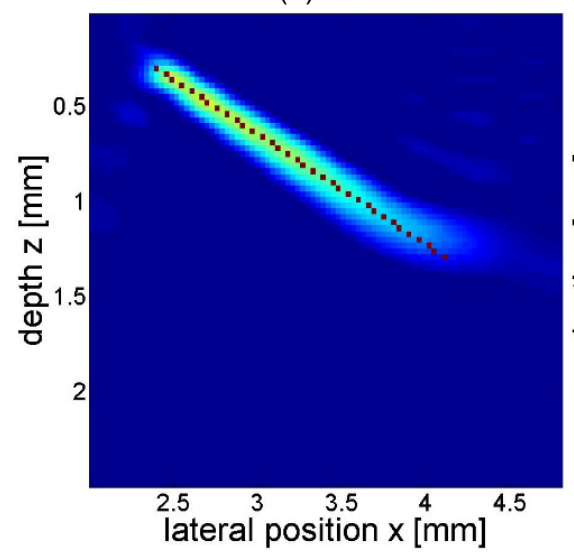

(b)

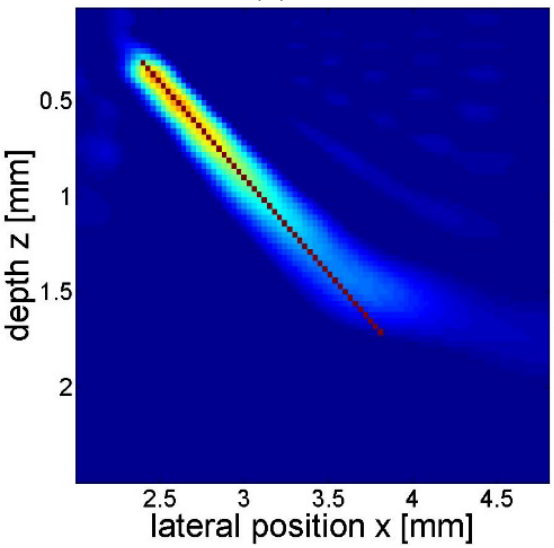

(c)

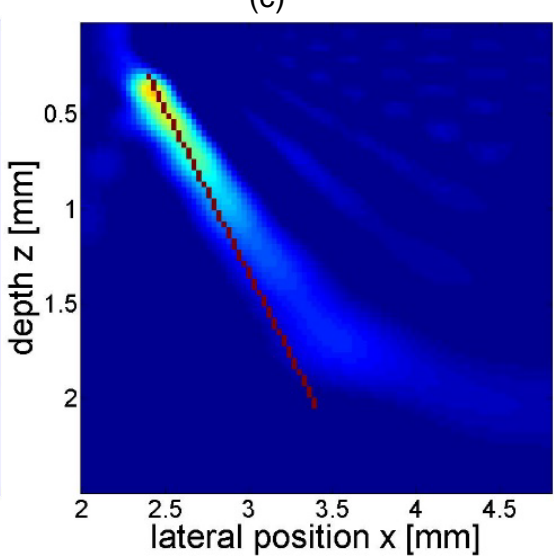

Fig. 2: $3 D$ reconstruction of a $2 \mathrm{~mm}$ inclined crack for: a) 30 degrees, b) 45 degrees and c) 60 degrees

The data received from the experimental testing was processed with the same reconstruction method. Instead of a simulated temperature evolution for each surface pixel, the IR camera data was used. These results have shown strong similarities to the results from the synthetic data as seen in the previous chapter (Fig.2). 


\section{Acknowledgement}

This work was supported by the "K-Project for non-destructive testing and tomography plus" supported by COMET program of FFG and the federal government of Upper Austria and Styria and by the project "multimodal and insitu characterization of inhomogeneous materials" (MiCi) by the federal government of Upper Austria and the European Regional Development Fund (EFRE) in the framework of the EU-program IWB2020. We also acknowledge funding from the federal government of Upper Austria within the strategic-economic research program "Innovative Upper Austria 2020".

\section{REFERENCES}

[1] Burgholzer P., Gruber J.and G. Mayr. Three-dimensional thermographic imaging using a virtual wave concept. Journal of Applied Pyhsics. 2017;121:105-102.

[2] Ibarra-Castanedo C., Piau J.-M., Guilbert S., Avdelidis N. P., Genest M., Bendada A. and Maldague X. P. Comparative study of active thermography techniques for the nondestructive evaluation of honeycomb structures. Research in Nondestructive Evaluation. 2009;20:1-31.

[3] C. Ibarra-Castanedo and X. Maldague. Pulsed phase thermography reviewed. Quantitative InfraRed Thermography Journal, 2004;1:47-70.

[4] Balageas D. L., Roche J.-M., Leroy F.-H., Lui W.-M. and Gorbach A. M. The thermographic signal reconstruction method: a powerful tool for the enhancement of transient thermographic images. Biocybern Biomed Eng. 2015;35:1-9.

[5] Ibarra-Castanedo C., Gonzalez D. A., Galmiche F., Bendada A. and Maldague X. P. On signal transforms applied to pulsed thermography. Recent Res. Devel. Applied Phys. 2009;91-27. 